\title{
Interacting entropy-corrected new agegraphic dark energy in Brans-Dicke cosmology
}

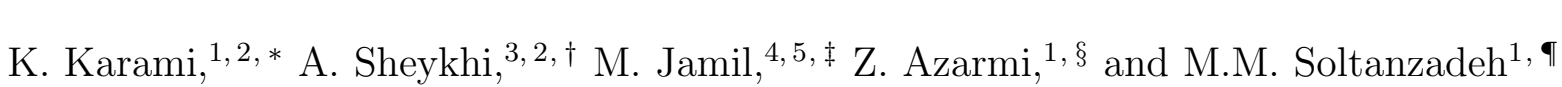 \\ ${ }^{1}$ Department of Physics, University of Kurdistan, Pasdaran St., Sanandaj, Iran \\ ${ }^{2}$ Research Institute for Astronomy \& Astrophysics of Maragha (RIAAM), Maragha, Iran \\ ${ }^{3}$ Department of Physics, Shahid Bahonar University, P.O. Box 76175, Kerman, Iran \\ ${ }^{4}$ Center for Advanced Mathematics and Physics (CAMP), \\ National University of Sciences and Technology (NUST), Islamabad, Pakistan \\ ${ }^{5}$ The Abdus Salam International Center for Theoretical \\ Physics (ICTP), Strada Costiera 11, Trieste, Italy
}

(Dated: October 16, 2018)

\begin{abstract}
Motivated by a recent work of one of us [1], we extend it by using quantum (or entropy) corrected new agegraphic dark energy in the Brans-Dicke cosmology. The correction terms are motivated from the loop quantum gravity which is one of the competitive theories of quantum gravity. Taking the non-flat background spacetime along with the conformal age of the universe as the length scale, we derive the dynamical equation of state of dark energy and the deceleration parameter. An important consequence of this study is the phantom divide scenario with entropycorrected new agegraphic dark energy. Moreover, we assume a system of dark matter, radiation and dark energy, while the later interacts only with dark matter. We obtain some essential expressions related with dark energy dynamics. The cosmic coincidence problem is also resolved in our model.
\end{abstract}

*Electronic address: KKarami@uok.ac.ir

${ }^{\dagger}$ Electronic address: sheykhi@mail.uk.ac.ir

${ }^{\ddagger}$ Electronic address: mjamil@camp.nust.edu.pk

$\S$ Electronic address: azarmi z@yahoo.com

『Electronic address: msoltanzadeh@uok.ac.ir 


\section{INTRODUCTION}

The present acceleration of the universe expansion has been well established through numerous and complementary cosmological observations [2]. A component which is responsible for this accelerated expansion usually dubbed "dark energy" (DE). The combined analysis of astronomical observations indicate that nearly three quarters of the universe consists of DE with negative pressure. Up to now different DE models have been proposed to explain this acceleration while most of them cannot explain all the features of universe or they have so many parameters, making them difficult to fit. The dynamical nature of DE, at least in an effective level, can originate from various fields, although a complete description requires a deeper understanding of the underlying theory of quantum gravity. Nevertheless, physicists can still make some attempts to probe the nature of DE according to some basic quantum gravitational principles. Two examples of such a paradigm are the holographic DE (HDE) and the agegraphic DE (ADE) models which are originated and possess some significant features of quantum gravity. The former, that arose a lot of enthusiasm recently [3 6], is motivated from the holographic hypothesis [7] and has been tested and constrained by various astronomical observations [8]. The later is originated form uncertainty relation of quantum mechanics together with the gravitational effect in general relativity. The ADE model assumes that the observed DE comes from the spacetime and matter field fluctuations in the universe. Following the line of quantum fluctuations of spacetime, Karolyhazy et al. [9] discussed that the distance $t$ in Minkowski spacetime cannot be known to a better accuracy than $\delta t=\lambda t_{P}^{2 / 3} t^{1 / 3}$ where $\lambda$ is a dimensionless constant of order unity and $t_{P}$ is the reduced Planck time. Based on Karolyhazy relation, Maziashvili [10] discussed that the energy density of metric fluctuations of the Minkowski spacetime is given by

$$
\rho_{\Lambda} \sim \frac{1}{t_{P}^{2} t^{2}} \sim \frac{M_{P}^{2}}{t^{2}},
$$

where $M_{P}$ is the reduced Planck mass $M_{P}^{-2}=8 \pi G$. Based on Karolyhazy relation [9] and Maziashvili arguments [10], Cai proposed the original ADE model to explain the accelerated expansion of the universe [11]. The original ADE has the following energy density [11]

$$
\rho_{\Lambda}=\frac{3 n^{2} M_{P}^{2}}{T^{2}}
$$


where $T$ is the age of the universe and given by

$$
T=\int \mathrm{d} t=\int_{0}^{a} \frac{\mathrm{d} a}{H a} .
$$

Also the numerical factor $3 n^{2}$ is introduced to parameterize some uncertainties, such as the species of quantum fields in the universe, the effect of curved spacetime (since the energy density is derived for Minkowski spacetime), and so on. However, the original ADE model had some difficulties. For example it suffers from the difficulty to describe the matterdominated epoch. Therefore, a new model of ADE was proposed by Wei and Cai [12], while the time scale is chosen to be the conformal time instead of the age of the universe. The energy density of the new ADE (NADE) is given by [12]

$$
\rho_{\Lambda}=\frac{3 n^{2} M_{P}^{2}}{\eta^{2}}
$$

where $\eta$ is conformal time of the FRW universe and given by

$$
\eta=\int \frac{\mathrm{d} t}{a}=\int_{0}^{a} \frac{\mathrm{d} a}{H a^{2}} .
$$

The NADE contains some new features different from the original ADE and overcome some unsatisfactory points. The ADE models have been examined and studied in ample detail 13 17].

It is worthy to note that the definition and derivation of HDE density depends on the entropy-area relationship of black holes in Einsteins gravity [3]. However, this definition can be modified from the inclusion of quantum effects, motivated from the loop quantum gravity (LQG). The quantum corrections provided to the entropy-area relationship leads to the curvature correction in the Einstein-Hilbert action and vice versa [18, 19]. The corrected entropy takes the form [20]

$$
S=\frac{A}{4 G}+\tilde{\alpha} \ln \frac{A}{4 G}+\tilde{\beta},
$$

where $\tilde{\alpha}$ and $\tilde{\beta}$ are dimensionless constants of order unity. These corrections arise in the black hole entropy in LQG due to thermal equilibrium fluctuations and quantum fluctuations [21]. Motivated by the corrected entropy-area relation (6), Wei [22] proposed the energy density of the so-called "entropy-corrected HDE" (ECHDE) as

$$
\rho_{\Lambda}=3 n^{2} M_{P}^{2} L^{-2}+\alpha L^{-4} \ln \left(M_{P}^{2} L^{2}\right)+\beta L^{-4},
$$

where $\alpha$ and $\beta$ are dimensionless constants of order unity. On the other hand, soon after "general theory of relativity" was introduced by Albert Einstein in 1915, several attempts 
to construct alternative theories of gravity were made. One of the most studied alternative theories was scalar-tensor theory, where the gravitational action contains, apart from the metric, a scalar field which describes part of the gravitational field. The scalar-tensor theory was invented first by Jordan [23] in the 1950's, and then taken over by Brans and Dicke [24] some years later. The starting point of the scalar-tensor theory sharing stage with gravitation is the idea of Mach. This theory has got a new impetus recently as it arises naturally as the low energy limit of many theories of quantum gravity such as superstring theory or KaluzaKlein theory [25]. Because the ADE density belongs to a dynamical cosmological constant, we need a dynamical frame to accommodate it instead of Einstein gravity. Therefore the investigation on the agegraphic models of DE in the framework of Brans-Dicke theory is of great importance. The investigation on the holographic/agegraphic models of DE in the framework of Brans-Dicke cosmology, have been carried out in [26 30].

In this paper we would like to study the so-called entropy-corrected NADE (ECNADE) whose $L$ in Eq. (17) is replaced with the conformal time $\eta$ of the universe. Therefore we propose the energy density of ECNADE of the form

$$
\rho_{\Lambda}=\frac{3 n^{2} M_{P}^{2}}{\eta^{2}}+\frac{\alpha}{\eta^{4}} \ln \left(M_{P}^{2} \eta^{2}\right)+\frac{\beta}{\eta^{4}} .
$$

In the special case $\alpha=\beta=0$, Eq. (8) yields the NADE density (41) in Einstein gravity [12]. Note that in Eq. (8) like the NADE density (4) to justify the matter-dominated era, the time scale is chosen to be the conformal time instead of the age of the universe.

The motivation idea for taking the energy density of modified NADE in the form (8) comes from the fact that both NADE and HDE models have the same origin. Indeed, it was argued that the NADE models are the HDE model with different IR length scales [31]. Since the last two terms in Eq. (8) can be comparable to the first term only when $\eta$ is very small, the corrections make sense only at the early stage of the universe. When the time scale $\eta$ becomes large, ECNADE reduces to the ordinary NADE. Although it is believed that our universe is flat, a contribution to the Friedmann equation from spatial curvature is still possible if the number of e-foldings is not very large (see Huang and Li in [3]). Besides, some experimental data has implied that our universe is not a perfectly flat universe and recent papers have favored the universe with spatial curvature [32]. 


\section{ECNADE IN BRANS-DICKE THEORY}

The action of four-dimensional Brans-Dicke theory in the canonical form can be written as [33]

$$
S=\int \mathrm{d}^{4} x \sqrt{g}\left(-\frac{1}{8 \omega} \phi^{2} R+\frac{1}{2} g^{\mu \nu} \partial_{\mu} \phi \partial_{\nu} \phi+L_{M}\right),
$$

where $R$ is the scalar curvature and $\phi$ is the Brans-Dicke scalar field. The non-minimal coupling term $\phi^{2} R$ replaces with the Einstein-Hilbert term $R / G$ in such a way that $G_{\text {eff }}^{-1}=$ $2 \pi \phi^{2} / \omega$ where $G_{\text {eff }}$ is the effective gravitational constant as long as the dynamical scalar field $\phi$ varies slowly. The signs of the non-minimal coupling term and the kinetic energy term are properly adopted to (+---) metric signature. The ECNADE model will be accommodated in the non-flat Friedmann-Robertson-Walker (FRW) universe which is described by the line element

$$
\mathrm{d} s^{2}=\mathrm{d} t^{2}-a^{2}(t)\left(\frac{\mathrm{d} r^{2}}{1-k r^{2}}+r^{2} \mathrm{~d} \Omega^{2}\right)
$$

where $a(t)$ is the scale factor, and $k$ is the curvature parameter with $k=-1,0,1$ corresponding to open, flat, and closed universes, respectively. A closed universe with a small positive curvature $\left(\Omega_{k} \simeq 0.02\right)$ is compatible with observations [32]. The field equations can be obtained by varying action (9) with respect to metric (10) which leads

$$
\begin{gathered}
\frac{3}{4 \omega} \phi^{2}\left(H^{2}+\frac{k}{a^{2}}\right)-\frac{1}{2} \dot{\phi}^{2}+\frac{3}{2 \omega} H \dot{\phi} \phi=\rho_{r}+\rho_{m}+\rho_{\Lambda}, \\
\frac{-1}{4 \omega} \phi^{2}\left(2 \frac{\ddot{a}}{a}+H^{2}+\frac{k}{a^{2}}\right)-\frac{1}{\omega} H \dot{\phi} \phi-\frac{1}{2 \omega} \ddot{\phi} \phi-\frac{1}{2}\left(1+\frac{1}{\omega}\right) \dot{\phi}^{2}=p_{r}+p_{\Lambda}, \\
\ddot{\phi}+3 H \dot{\phi}-\frac{3}{2 \omega}\left(\frac{\ddot{a}}{a}+H^{2}+\frac{k}{a^{2}}\right) \phi=0,
\end{gathered}
$$

where the dot stands for the derivative with respect to time and $H=\dot{a} / a$ is the Hubble parameter. Here $\rho_{\Lambda}, p_{\Lambda}, \rho_{r}, p_{r}$ and $\rho_{m}$ are, respectively, the DE density, DE pressure, energy density of radiation, pressure of radiation and energy density of pressureless dust (dark matter). Here the contribution of radiation is also considered. Because according to Eq. (8), the last two terms make only sense at the early stage of the universe, i.e. the radiation-dominated epoch.

At this point our system of equations is not closed and we still have freedom to choose one. We shall assume that Brans-Dicke field can be described as a power law of the scale factor, $\phi \propto a^{\varepsilon}$. In principle there is no compelling reason for this choice. However, it has been shown that for small $\varepsilon$ it leads to consistent results [28, 30]. A case of particular interest 
is that when $\varepsilon$ is small whereas $\omega$ is high so that the product $\varepsilon \omega$ results of order unity [28]. This is interesting because local astronomical experiments set a very high lower bound on $\omega$; in particular, the Cassini experiment implies that $\omega>10^{4}[34]$. Taking the derivative with respect to time of relation $\phi \propto a^{\varepsilon}$, we get

$$
\begin{gathered}
\dot{\phi}=\varepsilon H \phi, \\
\ddot{\phi}=\varepsilon^{2} H^{2} \phi+\varepsilon \phi \dot{H} .
\end{gathered}
$$

In the framework of Brans-Dicke cosmology, we write down the energy density of the ECNADE model in the universe as

$$
\rho_{\Lambda}=\frac{3 n^{2} \phi^{2}}{4 \omega \eta^{2}}+\frac{\alpha}{\eta^{4}} \ln \left(\frac{\phi^{2} \eta^{2}}{4 \omega}\right)+\frac{\beta}{\eta^{4}}
$$

which can be rewritten as

$$
\rho_{\Lambda}=\frac{3 n^{2} \phi^{2}}{4 \omega \eta^{2}} \gamma_{n}
$$

where

$$
\gamma_{n}=1+\frac{4 \omega \alpha}{3 n^{2} \phi^{2} \eta^{2}} \ln \left(\frac{\phi^{2} \eta^{2}}{4 \omega}\right)+\frac{4 \omega \beta}{3 n^{2} \phi^{2} \eta^{2}},
$$

shows the deviation from the NADE model. In the above equation $\phi^{2}=\omega / 2 \pi G_{\text {eff }}$. In the limiting case $G_{\text {eff }} \rightarrow G$, expression (16) restores the energy density of ECNADE in Einstein gravity [17]. The critical energy density, $\rho_{\mathrm{cr}}$, and the energy density of the curvature, $\rho_{k}$, are defined as

$$
\rho_{\mathrm{cr}}=\frac{3 \phi^{2} H^{2}}{4 \omega}, \quad \rho_{k}=\frac{3 k \phi^{2}}{4 \omega a^{2}} .
$$

The fractional energy densities are also defined as usual

$$
\Omega_{r}=\frac{\rho_{r}}{\rho_{\mathrm{cr}}}=\frac{4 \omega \rho_{r}}{3 \phi^{2} H^{2}}, \quad \Omega_{m}=\frac{\rho_{m}}{\rho_{\mathrm{cr}}}=\frac{4 \omega \rho_{m}}{3 \phi^{2} H^{2}}, \quad \Omega_{k}=\frac{\rho_{k}}{\rho_{\mathrm{cr}}}=\frac{k}{H^{2} a^{2}}, \quad \Omega_{\Lambda}=\frac{\rho_{\Lambda}}{\rho_{\mathrm{cr}}}=\frac{n^{2}}{H^{2} \eta^{2}} \gamma_{n} .
$$

\section{A. Noninteracting case}

Consider the FRW universe filled with ECNADE, pressureless matter and radiation which evolves according to their conservation laws

$$
\begin{gathered}
\dot{\rho}_{\Lambda}+3 H\left(1+w_{\Lambda}\right) \rho_{\Lambda}=0, \\
\dot{\rho}_{m}+3 H \rho_{m}=0,
\end{gathered}
$$




$$
\dot{\rho}_{r}+4 H \rho_{r}=0,
$$

where $w_{\Lambda}=p_{\Lambda} / \rho_{\Lambda}$ is the equation of state (EoS) parameter of ECNADE. Taking the derivative of Eq. (17) with respect to the cosmic time and using Eqs. (14), (20) and $\dot{\eta}=1 / a$ we have

$$
\dot{\rho}_{\Lambda}=2 H \rho_{\Lambda}\left\{\frac{\varepsilon}{\gamma_{n}}+\frac{1}{n a}\left(\frac{1}{\gamma_{n}}-2\right)\left(\frac{\Omega_{\Lambda}}{\gamma_{n}}\right)^{1 / 2}+\frac{4 \alpha \omega H^{2}}{3 n^{4} \phi^{2}} \frac{\Omega_{\Lambda}}{\gamma_{n}^{2}}\left[\varepsilon+\frac{1}{n a}\left(\frac{\Omega_{\Lambda}}{\gamma_{n}}\right)^{1 / 2}\right]\right\} .
$$

Inserting this equation in the conservation law (21), we obtain the EoS parameter of ECNADE model in the framework of Brans-Dicke theory

$$
w_{\Lambda}=-1-\frac{2 \varepsilon}{3} \frac{1}{\gamma_{n}}+\frac{2}{3 n a}\left(2-\frac{1}{\gamma_{n}}\right)\left(\frac{\Omega_{\Lambda}}{\gamma_{n}}\right)^{1 / 2}-\frac{8 \alpha \omega H^{2}}{9 n^{4} \phi^{2}} \frac{\Omega_{\Lambda}}{\gamma_{n}^{2}}\left[\varepsilon+\frac{1}{n a}\left(\frac{\Omega_{\Lambda}}{\gamma_{n}}\right)^{1 / 2}\right] .
$$

In the special case $\alpha=\beta=0$, from Eq. (18) we have $\gamma_{n}=1$ and Eq. (25) in the absence of radiation restores the EoS parameter of NADE in Brans-Dicke theory [1]

$$
w_{\Lambda}=-1-\frac{2 \varepsilon}{3}+\frac{2}{3 n a} \sqrt{\Omega_{\Lambda}} .
$$

Comparing Eq. (25) with (26) we see that in the presence of correction terms the scalar field $\phi$ enters the EoS parameter explicitly. From Eqs. (16) and (25) we see that in the late time where $\Omega_{\Lambda} \rightarrow 1$ and $a \rightarrow \infty$ we can neglect the last two terms in Eq. (25) and we find $w_{\Lambda}=-1-\frac{2 \varepsilon}{3 \gamma_{n}}$. Thus in the late time universe, although the EoS parameter of ECNADE does not feel the presence of the last two correction terms in Eq. (8) but for $\varepsilon \neq 0$ it will necessary cross the phantom divide, i.e. $w_{\Lambda}<-1$ in Brans-Dicke theory. This is in contrast to Einstein gravity $(\varepsilon \rightarrow 0)$ where $w_{\Lambda}$ of ECNADE mimics a cosmological constant in the late time [17].

Since in our model the dynamics of the scale factor is governed not only by the dark matter (DM), the radiation and the NADE, but also by the Brans-Dicke field, the signature of the deceleration parameter,

$$
q=-\frac{\ddot{a}}{a H^{2}}=-1-\frac{\dot{H}}{H^{2}},
$$

has to be examined carefully. When deceleration parameter is combined with the Hubble parameter and the dimensionless density parameters, form a set of useful parameters for the description of the astrophysical observations. Dividing Eq. (12) by $H^{2}$, and using Eqs. (14), (15), (16) and (201) we obtain

$$
q=\frac{1}{2 \varepsilon+2}\left[(2 \varepsilon+1)^{2}+2 \varepsilon(\varepsilon \omega-1)+\Omega_{k}+3 \Omega_{\Lambda} w_{\Lambda}+\Omega_{r}\right],
$$


where $w_{\Lambda}$ is given by Eq. (25).

We can also obtain the equation of motion for $\Omega_{\Lambda}$. Taking the derivative of the last Eq. (20) and using relation $\dot{\Omega}_{\Lambda}=H \Omega_{\Lambda}^{\prime}$, we obtain

$$
\Omega_{\Lambda}^{\prime}=\Omega_{\Lambda}\left[-\frac{2 \dot{H}}{H^{2}}-\frac{2}{n a}\left(\frac{\Omega_{\Lambda}}{\gamma_{n}}\right)^{1 / 2}+\frac{1}{H} \frac{\dot{\gamma_{n}}}{\gamma_{n}}\right]
$$

where the prime denotes the derivative with respect to $x=\ln a$. Finally, using Eqs. (14), (18), (20), (27) and $\dot{\eta}=1 / a$ we obtain

$$
\Omega_{\Lambda}^{\prime}=2 \Omega_{\Lambda}\left\{1+q-\frac{1}{n a}\left(\frac{\Omega_{\Lambda}}{\gamma_{n}}\right)^{1 / 2}+\left(\frac{1-\gamma_{n}}{\gamma_{n}}+\frac{4 \omega \alpha H^{2}}{3 n^{4} \phi^{2}} \frac{\Omega_{\Lambda}}{\gamma_{n}^{2}}\right)\left[\varepsilon+\frac{1}{n a}\left(\frac{\Omega_{\Lambda}}{\gamma_{n}}\right)^{1 / 2}\right]\right\} .
$$

\section{B. Interacting case}

Our aim here is to construct a cosmological model based on the Brans-Dicke theory of gravity and on the assumption that the pressureless DM and ECNADE do not conserve separately but interact with each other. Since we know neither the nature of DE nor the nature of DM, a microphysical interaction model is not available either. However, pressureless DM in interaction with DE is more reasonable than just another model to describe an accelerated expansion of the universe. Indeed, this possibility is receiving growing attention in the literature [35] and appears to be compatible with SNIa and CMB data [36]. Interaction causes the ECNADE and DM do not conserve separately and they must rather enter the energy balances

$$
\begin{gathered}
\dot{\rho}_{\Lambda}+3 H\left(1+w_{\Lambda}\right) \rho_{\Lambda}=-Q, \\
\dot{\rho}_{m}+3 H \rho_{m}=Q, \\
\dot{\rho}_{r}+4 H \rho_{r}=0,
\end{gathered}
$$

where we have assumed the radiation dose not interact with DE. Here $Q$ is an interaction term which can be an arbitrary function of cosmological parameters like the Hubble parameter and energy densities $Q\left(H \rho_{\Lambda}, H \rho_{m}\right)$. The dynamics of interacting DE models with different $Q$-classes have been studied in ample detail by [37]. It should be noted that the ideal interaction term must be motivated from the theory of quantum gravity. In the absence of such a theory, we rely on pure dimensional basis for choosing an interaction $Q$. Hence following [38], we assume $Q=\Gamma \rho_{\Lambda}$ with $\Gamma=3 b^{2}(1+u) H$ where $u=\rho_{m} / \rho_{\Lambda}$ and $b^{2}$ is a 
coupling constant. Note that $\Gamma>0$ shows that there is an energy transfer from the DE to DM. This expression for the interaction term $Q$ was first introduced in the study of the suitable coupling between a quintessence scalar field and a pressureless DM field [39].

Using Eqs. (14) and (19), we can rewrite the first Friedmann equation (11) as

$$
\rho_{\text {cr }}+\rho_{k}=\rho_{r}+\rho_{m}+\rho_{\Lambda}+\rho_{\phi},
$$

where we have defined

$$
\rho_{\phi}=\frac{1}{2} \varepsilon H^{2} \phi^{2}\left(\varepsilon-\frac{3}{\omega}\right) .
$$

Dividing Eq. (34) by $\rho_{\text {cr }}$, this equation further can be rewritten as

$$
\Omega_{r}+\Omega_{m}+\Omega_{\Lambda}+\Omega_{\phi}=1+\Omega_{k},
$$

where

$$
\Omega_{\phi}=\frac{\rho_{\phi}}{\rho_{\mathrm{cr}}}=-2 \varepsilon\left(1-\frac{\varepsilon \omega}{3}\right) .
$$

Therefore, the interaction term $Q$ can be expressed as

$$
Q=3 b^{2} H \rho_{\Lambda}\left[\frac{1+\Omega_{k}-\Omega_{r}+2 \varepsilon\left(1-\frac{\varepsilon \omega}{3}\right)}{\Omega_{\Lambda}}\right] .
$$

Finally, inserting Eqs. (24) and (38) in Eq. (31) we find the EoS parameter for the interacting ECNADE model in the framework of Brans-Dicke theory

$$
\begin{array}{r}
w_{\Lambda}=-1-\frac{2 \varepsilon}{3} \frac{1}{\gamma_{n}}+\frac{2}{3 n a}\left(2-\frac{1}{\gamma_{n}}\right)\left(\frac{\Omega_{\Lambda}}{\gamma_{n}}\right)^{1 / 2}-\frac{8 \alpha \omega H^{2}}{9 n^{4} \phi^{2}} \frac{\Omega_{\Lambda}}{\gamma_{n}^{2}}\left[\varepsilon+\frac{1}{n a}\left(\frac{\Omega_{\Lambda}}{\gamma_{n}}\right)^{1 / 2}\right] \\
-b^{2} \Omega_{\Lambda}^{-1}\left[1+\Omega_{k}-\Omega_{r}+2 \varepsilon\left(1-\frac{\varepsilon \omega}{3}\right)\right] .
\end{array}
$$

Comparing Eq. (39) with (25) shows that in the presence of interaction since the last expression in Eq. (39) has a negative contribution, hence crossing the phantom divide, i.e. $w_{\Lambda}<-1$, can be more easily achieved for than when the interaction between DE and DM is not considered.

In the absence of correction terms $(\alpha=\beta=0)$, from Eq. (18) we have $\gamma_{n}=1$ and Eq. (39) in the absence of radiation recovers the EoS parameter of interacting NADE in Brans-Dicke theory [1]

$$
w_{\Lambda}=-1-\frac{2 \varepsilon}{3}+\frac{2}{3 n a} \sqrt{\Omega_{\Lambda}}-b^{2} \Omega_{\Lambda}^{-1}\left[1+\Omega_{k}+2 \varepsilon\left(1-\frac{\varepsilon \omega}{3}\right)\right] .
$$


On the other hand, when $\varepsilon=0(\omega \rightarrow \infty)$ the Brans-Dicke scalar field becomes trivial, i.e. $\phi^{2}=\omega / 2 \pi G=4 \omega M_{P}^{2}$, and Eq. (39) in the absence of radiation reduces to its respective expression in ECNADE model in Einstein gravity [17]

$$
w_{\Lambda}=-1+\frac{2}{3 n a}\left(2-\frac{1}{\gamma_{n}}-\frac{\alpha H^{2}}{3 M_{P}^{2} n^{4}} \frac{\Omega_{\Lambda}}{\gamma_{n}^{2}}\right)\left(\frac{\Omega_{\Lambda}}{\gamma_{n}}\right)^{1 / 2}-b^{2}\left(\frac{1+\Omega_{k}}{\Omega_{\Lambda}}\right) .
$$

If we compare Eq. (39) with Eq. (41) we find out that when ECNADE is combined with Brans-Dicke field the transition from normal state where $w_{\Lambda}>-1$ to the phantom regime where $w_{\Lambda}<-1$ for the EoS of interacting DE can be more easily achieved for than when resort to the Einstein field equations is made.

The deceleration parameter $q$ is still obtained according to Eq. (28), where $w_{\Lambda}$ is now given by Eq. (39). Also the equation of motion for $\Omega_{\Lambda}$ takes the form (30), where $q$ is the deceleration parameter for the interacting case.

In the end, we would also comment on the resolution of cosmic coincidence problem, namely, why are the DM and DE densities of precisely the same order today? In other words, why the ratio of the two energy densities is of order unity i.e. $u \equiv \rho_{m} / \rho_{\Lambda} \simeq u_{0}$ ? (where $u_{0}$ is a finite constant of order unity) To show this, we follow the procedure given in [40]. Differentiating $u$ w.r.t. $t$ yields

$$
\dot{u}=3 H u\left[w_{\Lambda}+\frac{1+u}{u} \frac{\Gamma}{3 H}\right] \equiv f(u) .
$$

To find the critical point, we put $f(u)=0$ to get

$$
u_{c}=-\frac{\frac{\Gamma}{3 H}}{w_{\Lambda}+\frac{\Gamma}{3 H}} .
$$

Now the cosmic coincidence problem is alleviated if the given critical point is stable i.e. $f^{\prime}\left(u_{c}\right)<0$. In other words, a constraint is obtained on the state parameter

$$
w_{\Lambda}<-\frac{\Gamma}{3 H} \text {. }
$$

Therefore $\Gamma>0$ (refers to transfer of energy from DE to DM). We emphasize that this result holds independently to any form of DE and the gravity theory. Moreover the above result holds only if the background geometry is FRW. However to resolve the coincidence problem, we need to show that $u_{c}$ is of order unity. This will hold if $b^{2} \sim 1 / 4$ i.e. for a small but positive coupling parameter, which is also compatible with recent observational studies [41]. We would also comment that this problem is not resolved by other cosmological models (see e.g. [42]). 


\section{CONCLUSIONS}

Among various candidates to explain cosmic accelerated expansion, only NADE and HDE models are based on the entropy-area relation. This implies that the energy density of NADE depends on the length scales taken in the model. For different choices of length scales, the dynamics of NADE will be different. Consequently only those length scales are relevant to DE models which explain certain physical phenomena like DE dynamical state equation and phantom crossing. We have chosen the length scale to be the conformal age of the universe motivated by some previous studies. Note that the entropy-area relation depends on the gravity theory. When applying the curvature corrections to the gravity theory, it yields quantum corrections to the entropy-area relation. Consequently the definition of NADE acquires additional terms. These correction terms are important to the DE model when the chosen length scale is large or small. In the present model, small length scales yields new physics of the NADE.

We studied an interaction between DE and DM in the Brans-Dicke framework. The exact nature of this interaction is not understood due to our ignorance of the microphysics of both components. The interaction could be exotic and to be explained via beyond the standard model of particle physics theories. The prime motivation behind the assumed interaction is to resolve the cosmic-coincidence problem which asks why $\Omega_{m} \sim \Omega_{\Lambda}$ happens to be at present time? If the two energy densities match approximately, it suggests emphatically that the two components evolved not independently. In some recent studies, the radiation component also has been added to such interaction models to resolve the cosmic-triple-coincidence problem [43] with some new interesting dynamical and thermodynamical implications are observed.

In an earlier study [27], the author developed a correspondence between the HDE and the Brans-Dicke scalar field and showed that phantom divide is not possible in the Brans-Dicke gravity. However if the field is assumed to interact with matter (chameleon scalar field) then the phantom crossing is possible in the Brans-Dicke framework [44]. Another study [1] showed that phantom crossing is permissible in the Brans-Dicke framework if the HDE is replaced with the NADE. In the present paper, we have extended the later study by incorporating the correction terms in the NADE definition. An important consequence/prediction of the present model is that it allows the phantom crossing of the DE state parameter due to the presence of several free parameters. Note that the numerical values of parameters are 
not entirely arbitrary but are of order unity, consequently, sub-negative and super-negative values of $w_{\Lambda}$ are allowed for certain choices of these parameters. We emphasize that there is only a hint in some observational studies on the possible evolution of the equation of state parameter. Up to know, in spite of its shortcomings on the theoretical side, the $\Lambda$ CDM model appears observationally more solid than any other in the market [45].

\section{Acknowledgments}

The works of K. Karami and A. Sheykhi have been supported financially by Research Institute for Astronomy \& Astrophysics of Maragha (RIAAM), Maragha, Iran. M. Jamil would like to thank the kind hospitality of the Abdus Salam ICTP, Trieste, Italy where part of this work was completed. We would like to thank E. Abdalla and the referee for giving enlightening comments to improve this work.

[1] A. Sheykhi, Phys. Rev. D 81 (2010) 023525.

[2] A.G. Riess, et al., Astron. J. 116 (1998) 1009;

S. Perlmutter, et al., Astrophys. J. 517 (1999) 565;

P. de Bernardis, et al., Nature 404 (2000) 955;

S. Perlmutter, et al., Astrophys. J. 598 (2003) 102.

[3] A. Cohen, D. Kaplan, A. Nelson, Phys. Rev. Lett. 82 (1999) 4971;

S.D.H. Hsu, Phys. Lett. B 594 (2004) 13;

M. Li, Phys. Lett. B 603 (2004) 1;

Q.G. Huang, M. Li, JCAP 08 (2004) 013.

[4] M. Jamil, M.U. Farooq, M.A. Rashid, Eur. Phys. J. C 61 (2009) 471;

M. Jamil, E.N. Saridakis, M.R. Setare, Phys. Lett. B 679 (2009) 172;

M. Jamil, M.U. Farooq, Int. J. Theor. Phys. 49 (2010) 42;

M.R. Setare, M. Jamil, JCAP 02 (2010) 010.

[5] B. Wang, Y. Gong, E. Abdalla, Phys. Lett. B 624 (2005) 141;

B. Wang, C.Y. Lin, E. Abdalla, Phys. Lett. B 637 (2005) 357;

B. Wang, C.Y. Lin, D. Pavón, E. Abdalla, Phys. Lett. B 662 (2008) 1; 
A. Sheykhi, Class. Quantum Grav. 27 (2010) 025007.

[6] K. Karami, J. Fehri, Phys. Lett. B 684 (2010) 61;

K. Karami, J. Fehri, Int. J. Theor. Phys. 49 (2010) 1118;

K. Karami, A. Abdolmaleki, Phys. Scr. 81 (2010) 055901;

K. Karami, JCAP 01 (2010) 015;

K. Karami, arXiv:1002.0431.

[7] G. t Hooft, gr-qc/9310026;

L. Susskind, J. Math. Phys. 36 (1995) 6377.

[8] B. Feng, X. Wang, X. Zhang, Phys. Lett. B 607 (2005) 35;

X. Zhang, F.Q. Wu, Phys. Rev. D 72 (2005) 043524;

X. Zhang, F.Q. Wu, Phys. Rev. D 76 (2007) 023502.

[9] F. Karolyhazy, Nuovo. Cim. A 42 (1966) 390;

F. Karolyhazy, A. Frenkel, B. Lukacs, in Physics as natural Philosophy edited by A. Shimony,

H. Feschbach, MIT Press, Cambridge, MA, (1982);

F. Karolyhazy, A. Frenkel, B. Lukacs, in Quantum Concepts in Space and Time edited by R.

Penrose, C.J. Isham, Clarendon Press, Oxford, (1986).

[10] M. Maziashvili, Int. J. Mod. Phys. D 16 (2007) 1531;

M. Maziashvili, Phys. Lett. B 652 (2007) 165.

[11] R.G. Cai, Phys. Lett. B 657 (2007) 228.

[12] H. Wei, R.G. Cai, Phys. Lett. B 660 (2008) 113.

[13] K.Y. Kim, H.W. Lee, Y.S. Myung, Phys. Lett. B 660 (2008) 118;

H. Wei, R.G. Cai, Phys. Lett. B 663 (2008) 1;

J.P. Wu, D.Z. Ma, Y. Ling, Phys. Lett. B 663, (2008) 152;

Y.W. Kim, et al., Mod. Phys. Lett. A 23 (2008) 3049;

J. Zhang, X. Zhang, H. Liu, Eur. Phys. J. C 54 (2008) 303;

I.P. Neupane, Phys. Lett. B 673 (2009) 111;

M. Jamil, E.N. Saridakis, arXiv:1003.5637;

M. Jamil, A. Sheykhi, arXiv:1003.5043.

[14] A. Sheykhi, Phys. Lett. B 680 (2009) 113.

[15] A. Sheykhi, Int. J. Mod. Phys. D 18 (2009) 2023;

A. Sheykhi, Int. J. Mod. Phys. D 19 (2010) 305; 
A. Sheykhi, Phys. Lett. B 682 (2010) 329;

A. Sheykhi, arXiv:0909.0302.

[16] K. Karami, M.S. Khaledian, F. Felegary, Z. Azarmi, Phys. Lett. B 686 (2010) 216;

K. Karami, A. Abdolmaleki, Astrophys. Space Sci. (2010), DOI 10.1007/s10509-010-0362-6;

K. Karami, A. Abdolmaleki, arXiv:0909.2427.

[17] K. Karami, A. Sorouri, arXiv:1003.2365.

[18] T. Zhu, J.R. Ren, Eur. Phys. J. C 62 (2009) 413;

R.G. Cai, et al., Class. Quantum Grav. 26 (2009) 155018;

D.A. Easson, arXiv:1003.1528.

[19] M.R. Banerjee, S.K. Modak, JHEP 05 (2009) 063;

S.K. Modak, Phys. Lett. B 671 (2009) 167;

R. Banerjee, S. Gangopadhyay, S.K. Modak, Phys. Lett. B 686 (2010) 181.

[20] S. Nojiri, S.D. Odintsov, Int. J. Mod. Phys. A 16 (2001) 3273;

J. Lidsey, et al., Phys. Lett. B 544 (2002) 337;

R. Banerjee, B.R. Majhi, Phys. Lett. B 662 (2008) 62;

J. Zhang, Phys. Lett. B 668 (2008) 353;

R. Banerjee, B.R. Majhi, JHEP 06 (2008) 095;

R. Banerjee, B.R. Majhi, S. Samanta, Phys. Rev. D 77 (2008) 124035;

R. Banerjee, B.R. Majhi, Phys. Lett. B 674 (2009) 218;

B.R. Majhi, Phys. Rev. D 79 (2009) 044005;

M. Jamil, M.U. Farooq, JCAP 03 (2010) 001;

F. Caravelli, L. Modesto, arXiv:1001.4364;

L. Modesto, A. Randono, arXiv:1003.1998.

[21] C. Rovelli, Phys. Rev. Lett. 77 (1996) 3288;

A. Ashtekar, J. Baez, A. Corichi, K. Krasnov, Phys. Rev. Lett. 80 (1998) 904;

K.A. Meissner, Class. Quant. Grav. 21 (2004) 5245;

A.J.M. Medved, E.C. Vagenas, Phys. Rev. D 70 (2004) 124021;

A. Ghosh, P. Mitra, Phys. Rev. D 71 (2005) 027502.

[22] H. Wei, Commun. Theor. Phys. 52 (2009) 743.

[23] P. Jordan, Schwerkraft und Weltall, Friedrich Vieweg und Sohn, Braunschwig (1955).

[24] C. Brans, R.H. Dicke, Phys. Rev. 124 (1961) 925. 
[25] M.B. Green, J.H. Schwarz, E. Witten, Superstring Theory, Cambridge University Press, Cambridge (1987).

[26] Y. Gong, Phys. Rev. D 70 (2004) 064029;

H. Kim, H.W. Lee, Y.S. Myung, Phys. Lett. B 628 (2005) 11.

[27] M.R. Setare, Phys. Lett. B 644 (2007) 99.

[28] N. Banerjee, D. Pavón, Phys. Lett. B 647 (2007) 447.

[29] A. Sheykhi, Phys. Lett. B 681 (2009) 205.

[30] L. Xu, J. Lu, W. Li, arXiv:0905.4174.

[31] Y.S. Myung, M.G. Seo, Phys. Lett. B 671 (2009) 435.

[32] C.L. Bennett, et al., Astrophys. J. Suppl. 148 (2003) 1;

D.N. Spergel, Astrophys. J. Suppl. 148 (2003) 175;

U. Seljak, A. Slosar, P. McDonald, JCAP 10 (2006) 014;

D.N. Spergel, et al., Astrophys. J. Suppl. 170 (2007) 377.

[33] M. Arik, M.C. Calik, Mod. Phys. Lett. A 21 (2006) 1241.

[34] B. Bertotti, L. Iess, P. Tortora, Nature, 425 (2003) 374;

V. Acquaviva, L. Verde, JCAP 12 (2007) 001.

[35] L. Amendola, Phys. Rev. D 60 (1999) 043501;

L. Amendola, C. Quercellini, Phys. Rev. D 68 (2003) 023514;

K. Karami, S. Ghaffari, J. Fehri, Eur. Phys. J. C 64 (2009) 85;

K. Karami, S. Ghaffari, Phys. Lett. B 685 (2010) 115;

K. Karami, S. Ghaffari, Phys. Lett. B 688 (2010) 125.

[36] G. Olivares, F. Atrio, D. Pavón, Phys. Rev. D 71 (2005) 063523.

[37] M. Jamil, M.A. Rashid, Eur. Phys. J. C 56 (2008) 429;

G. Caldera-Cabral, R. Maartens, L.A. Ureña-López, Phys. Rev. D 79 (2009) 063518;

L.P. Chimento, Phys. Rev. D 81 (2010) 043525.

[38] H. Kim, H.W. Lee, Y.S. Myung, Phys. Lett. B 632 (2006) 605.

[39] L. Amendola, Phys. Rev. D 62 (2000) 043511;

W. Zimdahl, D. Pavón, L.P. Chimento, Phys. Lett. B 521 (2001) 133;

W. Zimdahl, D. Pavón, Gen. Rel. Grav. 35 (2003) 413;

L.P. Chimento, A.S. Jakubi, D. Pavón, W. Zimdahl, Phys. Rev. D 67 (2003) 083513.

[40] D. Pavón, W. Zimdahl, Phys. Lett. B 628 (2005) 206; 
T. Gonzalez, I. Quiros, Class. Quantum Gravit. 25 (2008) 175019.

[41] B. Wang et al, Nucl. Phys. B 778 (2007) 69;

J. Valiviita et al, Mon. Not. R. Astron. Soc. 402 (2010) 2355.

[42] S. del Campo et al, Phys. Rev. D 78 (2008) 021302(R);

S. del Campo et al, J. Cosmo. Astropart. Phys. 0901 (2009) 020.

[43] N. Cruz, S. Lepe, F. Pena, arXiv:0910.1307;

M. Jamil, F. Rahaman, M. Kalam, Eur. Phys. J. C 60 (2009) 149;

M. Jamil, F. Rahaman, Eur. Phys. J. C 64 (2009) 97;

M. Jamil, E.N. Saridakis, M.R. Setare, Phys. Rev. D 81 (2010) 023007.

[44] M.R. Setare, M. Jamil, Phys. Lett. B 690 (2010) 1.

[45] P. Serra, et al., Phys. Rev. D 80 (2009) 121302(R);

R. Amanullah, et al., arXiv:1004.1711. 